\title{
VARIATIONS IN THE PRACTISE OF THE INTERSCALENE BLOCK AMONGST UK ANAESTHETISTS
}

\author{
D Teszka ${ }^{1}$, O Peralta ${ }^{1}$, A Sardesai ${ }^{1}$ \\ ${ }^{1}$ Department of Anaesthesia, Cambridge University Hospitals NHS Foundation Trust, UK
}

\section{Background and Aims:}

There is significant variation in the practise of the interscalene block. A survey was conducted aimed at assessing both adherence to published guidelines for consent and to obtain a profile on the extent of variation in techniques.

\section{Method:}

The survey was distributed via email to anaesthetists of all grades at three large NHS trusts in the UK. Responses were collected anonymously and concerned consent, use of sedation/GA prior to the block, local anaesthetic volume, nerve localisation methods, and complications.

\section{Results:}

A diverse group of anaesthetists of different grades from a geographical area including the East of England and East Midlands completed the questionnaire, with a sample size of 53 respondents, mainly at the Consultant level (62\%). Consent was most often obtained on the day of the procedure (91\%) and risks were mainly communicated verbally, with an information leaflet being used on occasion (13\%). Risks classed as 'other', noted by respondents to include LA toxicity, intrathecal injection, and pneumothorax were rarely discussed. Of the available options, infection (57\%) and bleeding (55\%) were the least likely risks to be conveyed ${ }^{b}$ to the patient, whilst the possibility of failure was stated $98 \%$ of the time. Respondents indicated failure as the most common complication they observed in the past 5 years ${ }^{c}$ (36\%) with no complications reported by $45 \%$. The most common LA volume used was between 10 and $19 \mathrm{~mL}(62 \%)$ and ultrasound was used $94 \%$ of the time, with nerve stimulation only in $6 \%$. Preference in performing the block on an awake patient was reported by $62 \%$ of respondents, with the remainder under sedation (17\%) or GA $(21 \%)^{a}$.

\section{Discussion:}

These results indicate that there are variations in the practise of Interscalene block, particularly in that certain operators prefer an awake patient, and others proceed with sedation/GA. Benumof produced a case series ${ }^{1}$ which correlates GA as a contributing factor in four catastrophic cervical spinal injections. Further investigation is necessary to determine whether GA is correlated to an increased risk of complications, however in this limited survey no respondent reported any major neurological complications and most perform the block on awake patients.

\section{References:}

1. Benumof JL. Permanent loss of cervical spinal cord function associated with interscalene block performed under general anesthesia. Anesthesiology. 2000 Dec;93(6):1541-4.

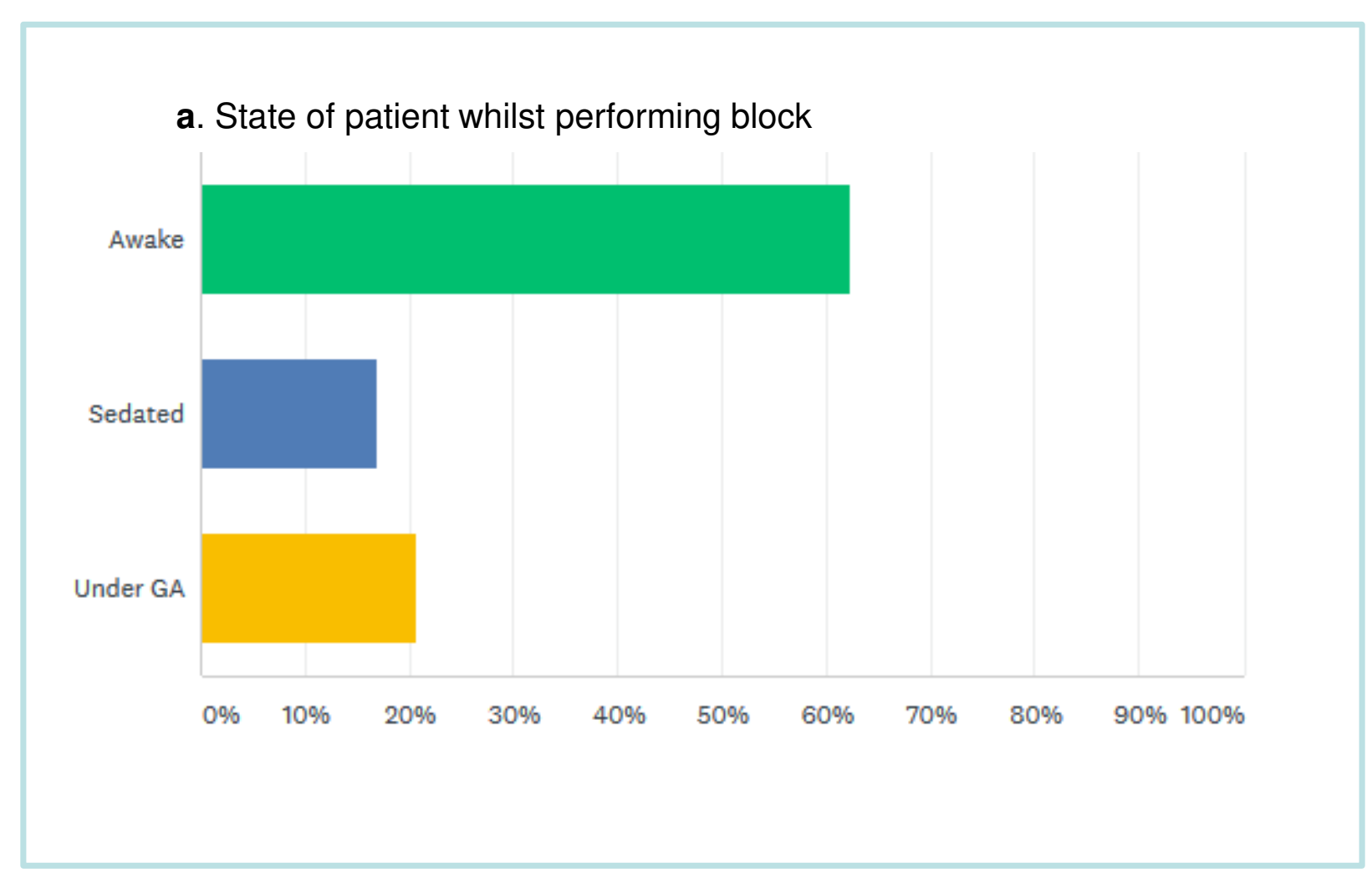

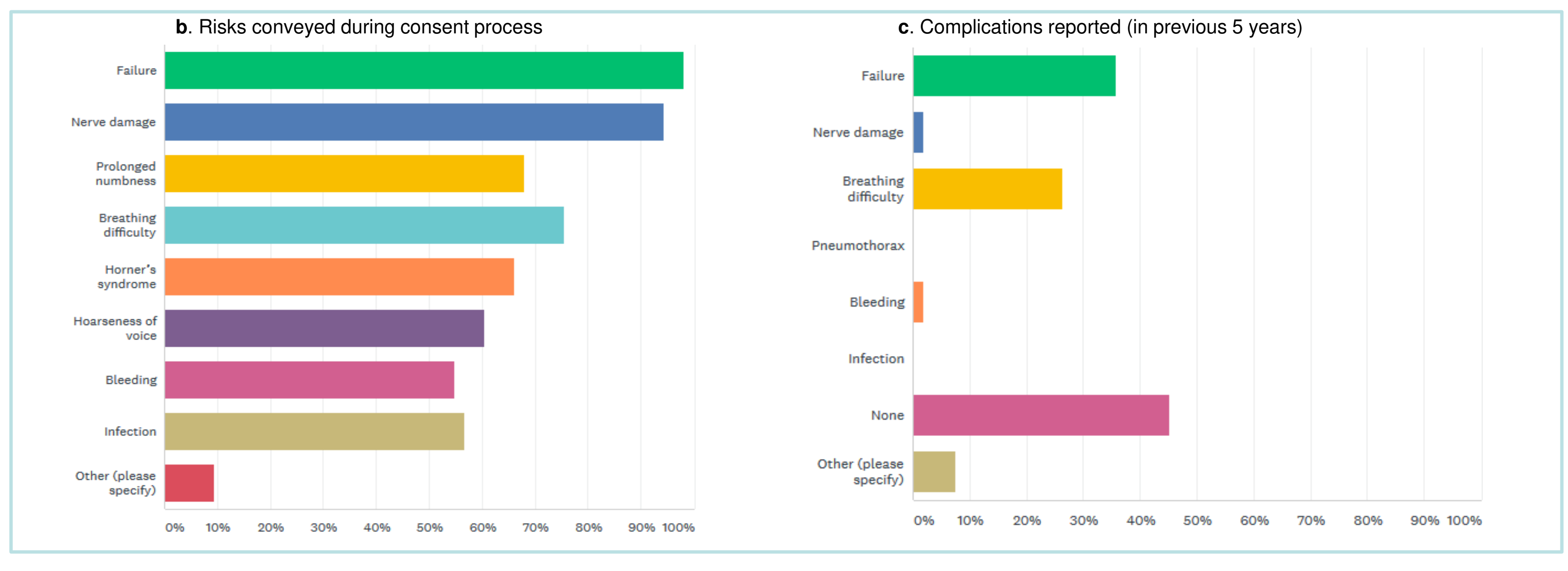

